\title{
A Study of Public Library Patrons' Understanding of Library Records and Data Privacy
}

\author{
Dong-Seok Kim*, Younghee Noh**
}

\author{
ARTICLE INFO \\ Article history: \\ Received 8 April 2014 \\ Revised 23 April 2014 \\ Accepted 1 June 2014 \\ Keywords: \\ Public Library, \\ User Privacy, \\ Personal Information, \\ Library Record, \\ Library Patron
}

\begin{abstract}
As instances of private information leak increase, taking steps to protect such information becomes a necessity. In this study of public library patrons, we strove for a comprehensive understanding of library usage records to suggest viable solutions for private information safety in public libraries. To this end, we investigated the patrons' understanding of library usage records and determined the relationship between different user characteristics and privacy knowledge or leaks. The results show that a high number of patrons perceived these records as their own private information, but that there was no necessity for legal procedures or consent for the use of these records. Also, even though the understanding of these usage records showed that there was a relationship between the frequency of library visits and leaks of personal information, the correlation was not particularly strong.
\end{abstract}

\section{Introduction}

Personal information, which became simple to collect and build due to the development of information technology, is being used to raise administration efficiency and as an important resource to create added value in public and private services. However, due to the rise in private information use and developments in technology, cases of illegal access and large-scale leaks are frequent.

In 2003 , there were 17,777 cases of infringement of personal data, which rose to 35,167 cases in 2009, 54,832 in 2010, 122,215 in 2011, and 166,801 in 2012, marking violation of private information as a serious and growing problem (Korea Internet and Security Agency, 2012).

Libraries are also using IT equipment to provide convenient services to patrons. However, with this convenience come problems, such as the exposure of patron private information and library usage records.

The United States has a long history of interest in protecting the private information of these library patrons. As far back as 1930, the 'Guidelines for Developing a Library Privacy Policy'

* Manager, Seogang Library of Mapo-Gu (aldebaran57@naver.com)

** Professor, Department of Library and Information Science, Konkuk University (irs4u@kku.ac.kr) (Corresponding Author)

International Journal of Knowledge Content Development \& Technology, 4(1): 53-78, 2014.

http://dx.doi.org/10.5865/IJKCT.2014.4.1.053 
proposed that it was the librarian's duty to keep all private information received private, and in 1995, libraries were urged to protect all patron records, including personal information and past borrowing history.

Here in Korea, the protection of private information of library patrons is also a priority, as shown in the Declaration of Library Ethics in 1997. As such, the Korean National Library tried to introduce a policy to guide libraries through processing private information in 2005 , although this policy suggestion did not have a large effect.

However, the Privacy Protection Act of 2011 provides a step-by-step protection standard for using, providing, and destroying private information. The act limits handling of personal information such as home address and other distinct identification information and also addresses the process for claims and requests to suspend the process of reading, changing, and deleting private information. These steps guarantee a better, stronger right for patrons that is guaranteed by the government (Son, 2012).

With this Act as a guide, the Ministry Of Culture established protection guidelines for private information and prescribed specific policies regarding different types of data, preventing violation of private information, and standards for those handling such information (Ministry of Culture, Sports and Tourism, 2012).

As evidenced by this attention, the protection of private information has become vital in all aspects of society. Therefore, libraries need to think carefully about how they are collecting, using, and storing patrons' private information and usage records.

In this study, we want to research the library patrons' private information and gain a comprehensive understanding of library usage records to suggest a management plan for the private information used in public libraries.

\section{Previous Research}

Kim (1994) suggested that privacy invasion prevention should involve privacy protection, user education, and statutory regulation and processes. Although most librarians agree that patrons' privacy must be protected, they are not fully aware of the different forms privacy violations might take.

Kang (2003) argued that even though most librarians understand that the patron's private data and loan records are information that should be protected, most institutes that have specific regulations or policies about this issue find that the same policies effect the rate of library use.

Kim and Nam (2004) argued that the library must guarantee that its patrons can easily access and use information. Specifically, in the study of the code of ethics for librarians, protection of one's private life is defined as keeping library usage records a secret and protecting the privacy of patrons, which is a point that needs a specific provision.

Noh (2012a) analyzed the possible privacy violations that could arise in libraries through the interference of other institutions (such as police or federal investigations), the process of providing library service, and the outsourcing of library tasks. In another study, Noh (2012b) argued that the accumulation of information for service personalization can also lead to privacy violations as well as other problems like filter bubbles. She clarified that a library is an institution that offers 
customized services tailored to its patrons, and, as such, there is the possibility of violating privacy and private information during library service. She suggested that further discussion on the protection of patron privacy is needed.

Enright (2001) argued that the laws, regulations, and guidelines regarding library private information policy must be clarified and revised, and suggested a clear checklist regarding this. Caldwell-Stone (2008) argued that protecting the patron's demand for information in a society where information is power is essential, and that it is the responsibility of the librarian to protect the privacy of library patrons.

In a study that suggested the library privacy protection policy, Lee (2005) examined the current state of information security in the network used in public libraries. With this data, he suggested maintenance supervision of Regional Central Library networks, forming public library privacy information protection guidelines and limited access, and further education on privacy protection for librarians, especially those in charge of data processing.

Kim (2006) studied the factors of the private information protection policy that are needed in college libraries and determined changes that college libraries needed, through analyzing domestic and foreign library private information protection policies and cases of violation.

Park (2010), in his study of patrons' guaranteed rights and invasion of private information, fully described the real condition of public institutes and libraries. He suggested private information protection guidelines for the private information protection in the library.

Noh (2012c), through the study of library private information protection policy, research on privacy violation cases, and analysis of laws and guidelines relating to domestic private information, suggested a suitable private information policy for the library as well as presented a case for the importance of establishing a policy for such protection.

Struges (2002) argued that librarians and others writing such policies must examine whether the privacy policy is ethical, legal, and realistic, if the public interest is being served, and if it represents the person directly concerned.

Falk (2004) revealed that many libraries have a codified privacy protection policy and that there are not enough regulations to protect patrons from the violations of private information in regard to the electronic technology used to provide services to the patrons. Falk argued that in this privacy problem, describing the policy that protects the patrons influences the actions of the librarian.

In a study examining a library system where the patrons' information was protected by giving them the ability to opt-out, Chung (2000) was able to see that the public library usage records which were able to be opened and seen after proper jurisdiction requirements, were not being handled through the proper proceedings. He suggested the establishment of an institutional cooperation system of other libraries for the education and insurance of public library user privacy.

Lee (2004), in a study of library intellectual freedom, researched the declaration of intellectual freedom in advanced countries, the freedom of collection records, and the policies towards passwords of patrons. With this information, Lee argued that privacy rights are included inherently within the concept of the right to know, and through the guarantee of privacy and the right to know, the intellectual freedom of library patrons can be fully realized.

Kim (2012) said the record of borrowed books is information that can reveal the ideals and 
conscience of patrons, making it sensitive private information that is related to the patrons' privacy. Kim concluded that the establishment of different policies and revisions of library law are necessary to protect patrons' privacy.

From an information technology aspect, Balas (2001) argued that due to the advancement in technology, the privacy issues have become more complicated. Even though problems caused by the Internet and copyright issues have an influence on the librarian, privacy problems in the digital generation present greater challenges.

Fifarek (2002) argued that greater privacy problems were due to the introduction of new technology. Fifarek proposed to discuss a case of privacy failure and related laws and a checklist for the privacy protection of patrons.

Shuler (2004) argued that before the electronic era, privacy was not a serious problem. However, in the digital knowledge information era, it is impossible to preserve it fully in a library due to the demand for a specific electronic identification, regulation, and statistics

Butters (2007) mentioned the danger of using RFID in libraries. Butters pointed out that the RFID standard now has a weak point where it allows the act of invading privacy and digital compromise and reviewed a way to lessen the danger from the standard RFID.

Klinefelter (2007) said that customizable user service was possible due to the development of technology. Klinefelter handled the possible privacy invasion cases that could arrive in a library and argued that even though they provided information materials related to the interest of each patron (information on new journals, mail and email informing of newly-arrived books and ones that need returning), anonymity and control over their information was sacrificed to provide these services.

Zimerman (2009) argued that the development of information technology had lowered the level of privacy in society. Zimerman pointed out a specific case of a privacy problem and criticized problems with hackers seeking to collecte private information.

Through this previous research, it is obvious that protecting a patron's private information is an active concern both domestically and abroad. Most researchers and librarians are aware of the necessity of protecting the private information of library patrons.

While insisting on the importance of protecting this private information, researchers have proposed developments for policy, revisions of the enactment article, and checklists. However, few studies have concentrated on how the patron views library usage records. Even though the patron allowed the collection and use of part of their private information, the study was done with college library patrons, which represents a different audience and is inappropriate for application to the public library.

Using the private information that is collected, preserved, and used in public libraries and the patron understanding of library usage records. we are attempting to determine a path for development of a management plan for library private information.

\section{Research questions}

As privacy leaks and, therefore, the demand for privacy protection increase, it is important to investigate how library patrons think about the collection and preservation of their private information 
to devise a solution for protect their privacy. Specifically, research needs to focus on library usage records, since this personal data, unlike personal identification information, is unique to libraries.

In this study, the following research questions were set to determine what kind of relationship exists between personal characteristics and awareness of library usage records after measuring what patrons thought about the collection and preservation of private information.

- Research question 1: Is there a meaningful relationship between frequent visits to libraries and a greater awareness of library usage records?

- Research question 2: Is there a meaningful relationship between a patron's interest in privacy and greater understanding of library usage records?

- Research question 3: Is there a meaningful relationship between a patron's experience with damage caused by private information leaks and understanding of library usage record?

In order to complete the study in accordance with the research question above, we developed a survey to analyze the patron understanding of library usage records.

\section{Research design and methodology}

\subsection{Sampling method and data collection process}

In this survey, the target population was Eunpyeong-gu public library patrons. The Eunpyeong-gu public library, which is registered in the National Library Statistics System, has three separate branches: Eunpyeong Public Library, JeungSan Public Digital Library, and Eum Am Public Digital Library.

The survey was distributed to about $0.1 \%$ of Eunpyeong-gu Public Library users, or 196 patrons. It was conducted for a total of 27 days, from October 3 through October 29, 2013. All the surveys were collected and a total of 188 were analyzed excluding those that were left incomplete.

\subsection{Survey Contents}

The survey questions used in this study included 6 items regarding personal information and 11 items delving into patron understanding of library data usage. The survey questions regarding library usage records were taken from previous research, specifically Noh (2012c, 2013), and then modified and supplemented by the researcher for the purposes of this study.

Ultimately, the survey was developed with 4 survey areas and 17 survey entries. The questions were developed to gather a picture of the patron's understanding of the collection of library records, use of library record, and library record leaks. The contents of the survey and composition of the questions are shown in Table 1. 
D. S. Kim \& Y. H. Noh
58 International Journal of Knowledge Content Development \& Technology Vol.4, No.1, 53-78 (June, 2014)

Table 1. Survey Contents

\begin{tabular}{|c|c|c|}
\hline Classification & Measured Content & Source \\
\hline $\begin{array}{l}\text { Collection of library } \\
\text { usage records }\end{array}$ & $\begin{array}{l}\text { Understanding of the collection of library } \\
\text { usage record }\end{array}$ & $\begin{array}{l}\text { Noh (2012c, 2013) } \\
\text { Wallace (1988) } \\
\text { Clarke (1998) } \\
\text { Johnston (2000) } \\
\text { Leiserson (2002) } \\
\text { Shuler (2004) } \\
\text { Falk (2004) } \\
\text { Breeding (2006) } \\
\text { DeMarco (2006) } \\
\text { Levine (2006) } \\
\text { Kennedy (2006) } \\
\text { Klinefelter (2007) } \\
\text { Tripathi \& Tripathi (2010) }\end{array}$ \\
\hline $\begin{array}{l}\text { Collection of library } \\
\text { usage record }\end{array}$ & The amount of library usage records collected & $\begin{array}{l}\text { Noh Yeonghui (2012c, 2013) } \\
\text { Wallace (1988) } \\
\text { Clarke (1998) } \\
\text { Johnston (2000) } \\
\text { Leiserson (2002) } \\
\text { Shuler (2004) } \\
\text { Falk (2004) } \\
\text { Breeding (2006) } \\
\text { DeMarco (2006) } \\
\text { Levine (2006) } \\
\text { Kennedy (2006) } \\
\text { Klinefelter (2007) } \\
\text { Tripathi \& Tripathi (2010) }\end{array}$ \\
\hline $\begin{array}{l}\text { Collection of library } \\
\text { usage record }\end{array}$ & $\begin{array}{l}\text { Understanding of the collection and } \\
\text { preservation of library usage records }\end{array}$ & $\begin{array}{l}\text { Noh Yeonghui (2012c, 2013) } \\
\text { Wallace (1988) } \\
\text { Clarke (1998) } \\
\text { Johnston (2000) } \\
\text { Leiserson (2002) } \\
\text { Shuler (2004) } \\
\text { Falk (2004) } \\
\text { Breeding (2006) } \\
\text { DeMarco (2006) } \\
\text { Levine (2006) } \\
\text { Kennedy (2006) } \\
\text { Klinefelter (2007) } \\
\text { Tripathi \& Tripathi (2010) }\end{array}$ \\
\hline $\begin{array}{l}\text { Collection of library } \\
\text { usage record }\end{array}$ & $\begin{array}{l}\text { Understanding library usage records as one's } \\
\text { own private information }\end{array}$ & $\begin{array}{l}\text { Noh Yeonghui (2012c, 2013) } \\
\text { Wallace (1988) } \\
\text { Clarke (1998) } \\
\text { Johnston (2000) } \\
\text { Leiserson (2002) } \\
\text { Shuler (2004) } \\
\text { Falk (2004) } \\
\text { Breeding (2006) } \\
\text { DeMarco (2006) } \\
\text { Levine (2006) } \\
\text { Kennedy (2006) }\end{array}$ \\
\hline
\end{tabular}


D. S. Kim \& Y. H. Noh

\begin{tabular}{|c|c|c|}
\hline Classification & Measured Content & Source \\
\hline & & $\begin{array}{l}\text { Klinefelter }(2007) \\
\text { Tripathi \& Tripathi (2010) }\end{array}$ \\
\hline $\begin{array}{l}\text { Collection of library } \\
\text { usage record }\end{array}$ & $\begin{array}{l}\text { The importance of library usage records as } \\
\text { private information }\end{array}$ & $\begin{array}{l}\text { Noh Yeonghui (2012c, 2013) } \\
\text { Wallace (1988) } \\
\text { Clarke (1998) } \\
\text { Johnston (2000) } \\
\text { Leiserson (2002) } \\
\text { Shuler (2004) } \\
\text { Falk (2004) } \\
\text { Breeding (2006) } \\
\text { DeMarco (2006) } \\
\text { Levine (2006) } \\
\text { Kennedy (2006) } \\
\text { Klinefelter (2007) } \\
\text { Tripathi \& Tripathi (2010) }\end{array}$ \\
\hline $\begin{array}{l}\text { Collection of library } \\
\text { usage record }\end{array}$ & Reason for collecting library usage records & $\begin{array}{l}\text { Noh Yeonghui (2012c, 2013) } \\
\text { Wallace (1988) } \\
\text { Clarke (1998) } \\
\text { Johnston (2000) } \\
\text { Leiserson (2002) } \\
\text { Shuler (2004) } \\
\text { Falk (2004) } \\
\text { Breeding (2006) } \\
\text { DeMarco (2006) } \\
\text { Levine (2006) } \\
\text { Kennedy (2006) } \\
\text { Klinefelter (2007) } \\
\text { Tripathi \& Tripathi (2010) }\end{array}$ \\
\hline $\begin{array}{l}\text { Use of library usage } \\
\text { records }\end{array}$ & $\begin{array}{l}\text { The necessity of library usage in library } \\
\text { management. }\end{array}$ & $\begin{array}{l}\text { Noh Yeonghui (2012c, 2013) } \\
\text { Wallace (1988) } \\
\text { Clarke (1998) } \\
\text { Johnston (2000) } \\
\text { Leiserson (2002) } \\
\text { Shuler (2004) } \\
\text { Falk (2004) } \\
\text { Breeding (2006) } \\
\text { DeMarco (2006) } \\
\text { Levine (2006) } \\
\text { Kennedy (2006) } \\
\text { Klinefelter (2007) } \\
\text { Tripathi \& Tripathi (2010) }\end{array}$ \\
\hline $\begin{array}{l}\text { Use of library usage } \\
\text { record }\end{array}$ & $\begin{array}{l}\text { The necessity of user content when using } \\
\text { library usage records }\end{array}$ & $\begin{array}{l}\text { Noh Yeonghui }(2012 \mathrm{c}, 2013) \\
\text { Wallace (1988) } \\
\text { Clarke (1998) } \\
\text { Johnston }(2000) \\
\text { Leiserson (2002) } \\
\text { Shuler (2004) } \\
\text { Falk (2004) } \\
\text { Breeding (2006) } \\
\text { DeMarco (2006) } \\
\text { Levine (2006) }\end{array}$ \\
\hline
\end{tabular}


D. S. Kim \& Y. H. Noh
60 International Journal of Knowledge Content Development \& Technology Vol.4, No.1, 53-78 (June, 2014)

\begin{tabular}{|c|c|c|}
\hline Classification & Measured Content & Source \\
\hline & & $\begin{array}{l}\text { Kennedy (2006) } \\
\text { Klinefelter (2007) } \\
\text { Tripathi \& Tripathi (2010) }\end{array}$ \\
\hline $\begin{array}{l}\text { Use of library usage } \\
\text { record }\end{array}$ & $\begin{array}{l}\text { The necessity of legal procedures when library } \\
\text { usage records are demanded by outside parties } \\
\text { or } 3^{\text {rd }} \text { parties }\end{array}$ & $\begin{array}{l}\text { Noh Yeonghui (2012c, 2013) } \\
\text { Wallace (1988) } \\
\text { Clarke (1998) } \\
\text { Johnston (2000) } \\
\text { Leiserson (2002) } \\
\text { Shuler (2004) } \\
\text { Falk (2004) } \\
\text { Breeding (2006) } \\
\text { DeMarco (2006) } \\
\text { Levine (2006) } \\
\text { Kennedy (2006) } \\
\text { Klinefelter (2007) } \\
\text { Tripathi \& Tripathi (2010) }\end{array}$ \\
\hline $\begin{array}{l}\text { Library usage records } \\
\text { leaks }\end{array}$ & $\begin{array}{l}\text { Level of seriousness felt when library usage } \\
\text { records are leaked }\end{array}$ & $\begin{array}{l}\text { Noh Yeonghui (2012c, 2013) } \\
\text { Wallace (1988) } \\
\text { Clarke (1998) } \\
\text { Johnston (2000) } \\
\text { Leiserson (2002) } \\
\text { Shuler (2004) } \\
\text { Falk (2004) } \\
\text { Breeding (2006) } \\
\text { DeMarco (2006) } \\
\text { Levine (2006) } \\
\text { Kennedy (2006) } \\
\text { Klinefelter (2007) } \\
\text { Tripathi \& Tripathi (2010) }\end{array}$ \\
\hline Library usage record leak & Cause of library usage record leak & $\begin{array}{l}\text { Noh Yeonghui (2012c, 2013) } \\
\text { Wallace (1988) } \\
\text { Clarke (1998) } \\
\text { Johnston (2000) } \\
\text { Leiserson (2002) } \\
\text { Shuler (2004) } \\
\text { Falk (2004) } \\
\text { Breeding (2006) } \\
\text { DeMarco (2006) } \\
\text { Levine (2006) } \\
\text { Kennedy (2006) } \\
\text { Klinefelter (2007) } \\
\text { Tripathi \& Tripathi (2010) }\end{array}$ \\
\hline Demographic attributes & Age & \\
\hline demographics attribution & Sex & \\
\hline demographics attribution & Level of education & \\
\hline demographics attribution & Frequency of library visits & \\
\hline demographics attribution & Interest in privacy & \\
\hline demographics attribution & $\begin{array}{l}\text { Experience in damages due library usage } \\
\text { record leaks }\end{array}$ & \\
\hline
\end{tabular}




\section{Results}

\subsection{Demographic Characteristics}

Among the Eunpyeong-gu public library patrons who participated in the survey, $36.2 \%$ visited less than once a week, while $29.3 \%$ visited twice a week, $18.6 \%$ visited three times a week, $7.4 \%$ visited four times a week, and $8.5 \%$ of patrons visited more than 5 times a week (see Table 2). When asked whether they were interested in privacy issues, the majority of patrons $(80.9 \%)$ were interested, $14.9 \%$ chose neutral, and $4.2 \%$ were not interested (see Table 3 ).

When asked if they had ever been the victim of a private information leak, $85.1 \%$ had no such experience while $14.9 \%$ did have experience (See Table 4).

Table 2. Frequency of Library Use

\begin{tabular}{llllllllllll}
\hline $\begin{array}{l}\text { Less than } \\
\text { once a week }\end{array}$ & Twice a week & $\begin{array}{l}\text { Three times a } \\
\text { week }\end{array}$ & \multicolumn{2}{l}{$\begin{array}{l}\text { Four times } \\
\text { a week }\end{array}$} & \multicolumn{2}{l}{$\begin{array}{l}\text { More than five } \\
\text { times }\end{array}$} & Total \\
\hline Freq & $\%$ & Freq & $\%$ & Freq & $\%$ & Freq & $\%$ & Freq & $\%$ & Freq & $\%$ \\
\hline 68 & 36.2 & 55 & 29.3 & 35 & 18.6 & 14 & 7.4 & 16 & 8.5 & 188 & 100.0 \\
\hline
\end{tabular}

Table 3. Interest in privacy Issues

\begin{tabular}{|c|c|c|c|c|c|c|c|c|c|c|c|}
\hline \multicolumn{2}{|c|}{ Very uninterested } & \multicolumn{2}{|c|}{ Uninterested } & \multicolumn{2}{|c|}{ Neutral } & \multicolumn{2}{|c|}{ Interested } & \multicolumn{2}{|c|}{ Very interested } & \multicolumn{2}{|l|}{ Total } \\
\hline Freq & $\%$ & Freq & $\%$ & Freq & $\%$ & Freq & $\%$ & Freq & $\%$ & Freq & $\%$ \\
\hline 2 & 1 & 6 & 3.2 & 28 & 14.9 & 140 & 74.5 & 12 & 6.4 & 188 & 100.0 \\
\hline
\end{tabular}

Table 4. Experience as the Victim of a Privacy Leak

\begin{tabular}{llllll}
\hline No such experience & Experienced & \multicolumn{2}{l}{ Total } \\
\hline Freq & $\%$ & Freq & $\%$ & Freq & $\%$ \\
\hline 160 & 85.1 & 28 & 14.9 & 188 & 100.0 \\
\hline
\end{tabular}

\subsection{Awareness of library usage records}

\subsubsection{Awareness of the collection of library usage records}

To determine the patrons' level of awareness in regards to library usage records, survey participants were asked if they considered library use records to be their own private information. They were also asked the importance of each different type of usage record.

While $50.6 \%$ did not know that the library usage record was collected, a total of $27.6 \%$ of patrons knew that it was collected (See Table 5). Despite a majority of patrons being unaware of the records before this survey, $67.5 \%$ considered such records their own private information whereas $12.8 \%$ did not (see Table 6). 
Patrons were asked to rate the importance of various types of collected library data on a 5-point Likert scale. The results show that the material borrowing records were rated highest at 3.78, followed by Internet usage data (3.46), CCTV footage (3.36), reference services and civil complaint records (3.33), inter-library loan record (3.27), records of pictures and footage of events (3.10), accessing library community records (3.06), and desired materials selection records (3.02) (see Table 7).

Table 5. Previous Knowledge of Patron Library Records

\begin{tabular}{|c|c|c|c|c|c|c|c|c|c|c|c|}
\hline \multicolumn{2}{|c|}{ Had no clue } & \multicolumn{2}{|c|}{ Did not know } & \multicolumn{2}{|c|}{ Neutral } & \multicolumn{2}{|c|}{ Knew } & \multicolumn{2}{|c|}{ Knew well } & \multicolumn{2}{|c|}{ Total } \\
\hline Freq & $\%$ & Freq & $\%$ & Freq & $\%$ & Freq & $\%$ & Freq & $\%$ & Freq & $\%$ \\
\hline 5 & 2.7 & 90 & 47.9 & 41 & 21.8 & 52 & 27.6 & 0 & 0 & 188 & 100.0 \\
\hline
\end{tabular}

Table 6. Degree to which Patrons Consider Library Records Private

\begin{tabular}{llllllllllll}
\hline Not in the least & No & \multicolumn{3}{c}{ Neutral } & \multicolumn{2}{l}{ Yes } & \multicolumn{2}{l}{ Very much } & Total \\
\hline Freq & $\%$ & Freq & $\%$ & Freq & $\%$ & Freq & $\%$ & Freq & $\%$ & Freq & $\%$ \\
\hline 0 & 0 & 24 & 12.8 & 37 & 19.7 & 121 & 64.4 & 6 & 3.1 & 188 & 100.0 \\
\hline
\end{tabular}

Table 7. Importance of Library Record Types

\begin{tabular}{lll}
\hline Type of Record & Average & Standard deviation \\
\hline Material borrowing record & 3.78 & 0.987 \\
Internet usage record & 3.46 & 1.031 \\
Inter-library loan record & 3.27 & 1.022 \\
Reference service \& civil complaint record & 3.33 & 0.963 \\
Library community access record & 3.06 & 1.078 \\
Event picture and footage record & 3.10 & 1.016 \\
Desired book selection record & 3.02 & 1.084 \\
CCTV footage record & 3.36 & 1.053 \\
\hline
\end{tabular}

\subsubsection{Patron understanding of library usage records}

To determine how well patrons understand library usage records, the survey asked for their thoughts on the necessity of such records, user consent, and legal procedures in place for when records may be demanded by an outside party, such as the police.

When asked about the necessity of such records for effective library management, $70.2 \%$ of patrons said that keeping the records was needed while $2.1 \%$ disagreed (see Table 8 ). In regards to user consent, $79.8 \%$ of patrons said that it was needed before keeping records while $10.1 \%$ of patrons said that it was not (see Table 9).

In the case of library usage records being demanded by an outside party, $85.7 \%$ of patrons said that legal procedures would be necessary while $2.6 \%$ did not (see Table 10). 
D. S. Kim \& Y. H. Noh
International Journal of Knowledge Content Development \& Technology Vol.4, No.1, 53-78 (June, 2014)

Table 8. Necessity of Patron Records for Library Operation

\begin{tabular}{llllllllllll}
\hline Very & unnecessary & \multicolumn{2}{l}{ Unnecessary } & \multicolumn{2}{l}{ Neutral } & \multicolumn{2}{l}{ Necessary } & \multicolumn{2}{l}{ Very Necessary } & Total \\
\hline Freq & $\%$ & Freq & $\%$ & Freq & $\%$ & Freq & $\%$ & Freq & $\%$ & Freq & $\%$ \\
\hline 1 & 0.5 & 3 & 1.6 & 52 & 27.7 & 132 & 70.2 & 0 & 0 & 188 & 100.0 \\
\hline
\end{tabular}

Table 9. Necessity of Patron Consent for Personal Record Use

\begin{tabular}{|c|c|c|c|c|c|c|c|c|c|c|c|}
\hline Very & unnecessary & Unnec & ssary & Neutr & & Neces & & Very & Necessary & Total & \\
\hline Freq & $\%$ & Freq & $\%$ & Freq & $\%$ & Freq & $\%$ & Freq & $\%$ & Freq & $\%$ \\
\hline 0 & 0 & 19 & 10.1 & 19 & 10.1 & 137 & 72.9 & 13 & 6.9 & 188 & 100.0 \\
\hline
\end{tabular}

Table 10. Necessity of Legal Procedures for Library Records Requested by a Third Party

\begin{tabular}{llllllllllll}
\hline Very & unnecessary & \multicolumn{2}{l}{ Unnecessary } & \multicolumn{2}{l}{ Neutral } & \multicolumn{2}{l}{ Necessary } & \multicolumn{2}{l}{ Very Necessary } & Total \\
\hline Freq & $\%$ & Freq & $\%$ & Freq & $\%$ & Freq & $\%$ & Freq & $\%$ & Freq & $\%$ \\
\hline 0 & 0 & 5 & 2.6 & 22 & 11.7 & 146 & 77.7 & 15 & 8.0 & 188 & 100.0 \\
\hline
\end{tabular}

\subsubsection{Patron understanding of library usage record leaks}

In order to study the patrons' understanding of library usage record leaks, patrons were asked to rate how serious they felt such a leak would be and what they thought might be a possible cause of such leaks. Among the patrons who completed the survey, 53.2\% thought that a leak was 'serious' while $23.4 \%$ did not (see Table 11). When ranking possible causes of library usage record leaks, patrons considered a problem caused by the computer system to be the most likely cause (35.1\%), followed by collection of unnecessary information $(33.5 \%)$, information provided to third parties $(27.1 \%)$, librarian carelessness $(3.2 \%)$, and patron carelessness $(1.1 \%)$ (see Table 12 ).

Table 11. Seriousness of Library Record Information Leak

\begin{tabular}{llllllllllll}
\hline \multicolumn{2}{ll}{ Not serious } & \multicolumn{2}{l}{ Not Very Serious } & \multicolumn{2}{l}{ Neutral } & \multicolumn{2}{l}{ Serious } & \multicolumn{2}{l}{ Very serious } & \multicolumn{2}{l}{ Total } \\
\hline Freq & $\%$ & Freq & $\%$ & Freq & $\%$ & Freq & $\%$ & Freq & $\%$ & Freq & $\%$ \\
\hline 0 & 0 & 44 & 23.4 & 44 & 23.4 & 89 & 47.3 & 11 & 5.9 & 188 & 100.0 \\
\hline
\end{tabular}

Table 12. Likely Causes of Library Record Information Leak

\begin{tabular}{|c|c|c|c|c|c|c|c|c|c|c|c|}
\hline \multicolumn{2}{|c|}{$\begin{array}{l}\text { Librarian } \\
\text { carelessness }\end{array}$} & \multicolumn{2}{|c|}{$\begin{array}{l}\text { Computer } \\
\text { System }\end{array}$} & \multicolumn{2}{|c|}{$\begin{array}{l}\text { Patron } \\
\text { carelessness }\end{array}$} & \multicolumn{2}{|c|}{$\begin{array}{l}\text { Collection of } \\
\text { unnecessary } \\
\text { information }\end{array}$} & \multicolumn{2}{|c|}{$\begin{array}{l}\text { Information } \\
\text { provided to third } \\
\text { parties }\end{array}$} & \multicolumn{2}{|l|}{ Total } \\
\hline Freq & $\%$ & Freq & $\%$ & Freq & $\%$ & Freq & $\%$ & Freq & $\%$ & Freq & $\%$ \\
\hline 6 & 3.2 & 66 & 35.1 & 2 & 1.1 & 63 & 33.5 & 51 & 27.1 & 188 & 100.0 \\
\hline
\end{tabular}




\subsection{Patron understanding as compared to patron personal characteristics}

\subsubsection{Understanding of library usage records based on frequency of library use}

\section{1) Patron understanding of collection of library usage records}

We hypothesized that the frequency with which patrons used the library might have some effect on patrons' level of understanding of library records, given more frequent exposure to the topic. After gathering data, we determined that there was a meaningful relationship between these two aspects, as well as another between library use frequency and the belief that library usage records represent private information (See Table 13).

Table 13. Frequency of Library Visits and Record Collection Understanding

\begin{tabular}{|c|c|c|c|c|c|c|c|c|c|c|c|c|}
\hline \multirow{2}{*}{$\begin{array}{l}\text { Item } \\
\text { Item }\end{array}$} & \multirow{2}{*}{$\begin{array}{l}\text { Classification } \\
\text { Classification }\end{array}$} & \multicolumn{2}{|c|}{$\begin{array}{l}\text { Less than } \\
\text { once } \\
\text { a week }\end{array}$} & \multicolumn{2}{|c|}{$\begin{array}{l}\text { Twice } \\
\text { a week }\end{array}$} & \multicolumn{2}{|c|}{$\begin{array}{l}\text { Three } \\
\text { times a } \\
\text { week }\end{array}$} & \multicolumn{2}{|c|}{$\begin{array}{l}\text { Four } \\
\text { times } \\
\text { a week }\end{array}$} & \multicolumn{2}{|c|}{$\begin{array}{l}\text { More than } \\
\text { five times } \\
\text { a week }\end{array}$} & \multirow{2}{*}{$\begin{array}{l}\text { Fisher's } \\
\text { Exact test } \\
\text { Fisher's } \\
\text { Exact test }\end{array}$} \\
\hline & & Freq & $\%$ & Freq & $\%$ & Freq & $\%$ & Freq & $\%$ & Freq & $\%$ & \\
\hline $\begin{array}{l}\text { Understanding of } \\
\text { collection of library } \\
\text { usage records }\end{array}$ & $\begin{array}{l}\text { Did not know } \\
\text { at all }\end{array}$ & 2 & 2.5 & 0 & 0 & 1 & 2.9 & 1 & 7.1 & 1 & 6.3 & .000 \\
\hline $\begin{array}{l}\text { Understanding of } \\
\text { collection of library } \\
\text { usage record }\end{array}$ & Did not know & 37 & 45.7 & 26 & 47.3 & 14 & 40.0 & 5 & 35.7 & 8 & 50.0 & .000 \\
\hline $\begin{array}{l}\text { Understanding of } \\
\text { collection of library } \\
\text { usage record }\end{array}$ & Neutral & 13 & 16.0 & 14 & 25.5 & 10 & 28.6 & 3 & 21.4 & 1 & 6.3 & .000 \\
\hline $\begin{array}{l}\text { Understanding of } \\
\text { collection of library } \\
\text { usage record }\end{array}$ & Knew & 16 & 19.8 & 15 & 27.3 & 10 & 28.6 & 5 & 35.7 & 6 & 37.5 & .000 \\
\hline $\begin{array}{l}\text { Understanding of } \\
\text { collection of library } \\
\text { usage record }\end{array}$ & Knew well & 0 & 0 & 0 & 0 & 0 & 0 & 0 & 0 & 0 & 0 & .000 \\
\hline $\begin{array}{l}\text { Privacy of library } \\
\text { usage record }\end{array}$ & Very much no & 0 & 0 & 0 & 0 & 0 & 0 & 0 & 0 & 0 & 0 & .011 \\
\hline $\begin{array}{l}\text { Understanding of } \\
\text { private information on } \\
\text { library usage record }\end{array}$ & No & 5 & 6.2 & 7 & 12.7 & 6 & 17.1 & 2 & 14.3 & 4 & 25.0 & .011 \\
\hline $\begin{array}{l}\text { Understanding of } \\
\text { private information on } \\
\text { library usage record }\end{array}$ & Neutral & 15 & 18.5 & 11 & 20.0 & 7 & 20.0 & 2 & 14.3 & 2 & 12.5 & .011 \\
\hline $\begin{array}{l}\text { Understanding of } \\
\text { private information on } \\
\text { library usage record }\end{array}$ & Yes & 44 & 54.3 & 37 & 67.3 & 21 & 60.0 & 10 & 71.4 & 9 & 56.3 & .011 \\
\hline $\begin{array}{l}\text { Understanding of } \\
\text { private information on } \\
\text { library usage record }\end{array}$ & Very much yes & 4 & 4.9 & 0 & 0 & 1 & 2.9 & 0 & 0 & 1 & 6.3 & .011 \\
\hline
\end{tabular}




\section{2) Patron understanding of the usage of library usage record}

We also determined if the frequency of library visits had a meaningful relationship with understanding the use of library records. The data (as shown in Table 14) shows that frequency of library visits is tied with views such as the necessity of legal procedures for when records are demanded by third parties, the necessity of patron consent in keeping and using records, and the necessity of keeping library use records in general.

Table 14. Frequency of Library Visit and Record Usage Understanding

\begin{tabular}{|c|c|c|c|c|c|c|c|c|c|c|c|c|}
\hline \multirow{2}{*}{$\begin{array}{l}\text { Item } \\
\text { Item }\end{array}$} & \multirow{2}{*}{$\begin{array}{l}\text { Classification } \\
\text { Classification }\end{array}$} & \multicolumn{2}{|c|}{$\begin{array}{l}\text { Less than } \\
\text { once } \\
\text { a week }\end{array}$} & \multicolumn{2}{|c|}{$\begin{array}{l}\text { Twice } \\
\text { a week }\end{array}$} & \multicolumn{2}{|c|}{$\begin{array}{l}\text { Three } \\
\text { times } \\
\text { a week }\end{array}$} & \multicolumn{2}{|c|}{$\begin{array}{l}\text { Four } \\
\text { times } \\
\text { a week }\end{array}$} & \multicolumn{2}{|c|}{$\begin{array}{l}\text { More than } \\
\text { five times } \\
\text { a week }\end{array}$} & \multirow{2}{*}{$\begin{array}{l}\text { Fisher's } \\
\text { Exact test } \\
\text { Fisher's } \\
\text { Exact test }\end{array}$} \\
\hline & & Freq & $\%$ & Freq & $\%$ & Freq & $\%$ & Freq & $\%$ & Freq & $\%$ & \\
\hline $\begin{array}{l}\text { Necessity of } \\
\text { library usage } \\
\text { record for } \\
\text { management }\end{array}$ & $\begin{array}{l}\text { Very } \\
\text { unnecessary }\end{array}$ & 1 & 1.2 & 0 & 0 & 0 & 0 & 0 & 0 & 0 & 0 & .043 \\
\hline $\begin{array}{l}\text { Necessity of } \\
\text { library usage } \\
\text { record in } \\
\text { management }\end{array}$ & Unnecessary & 2 & 2.5 & 0 & 0 & 0 & 0 & 0 & 0 & 1 & 6.3 & .043 \\
\hline $\begin{array}{l}\text { Necessity of } \\
\text { library usage } \\
\text { record in } \\
\text { management }\end{array}$ & Neutral & 15 & 18.5 & 16 & 29.1 & 12 & 34.3 & 4 & 28.6 & 5 & 31.3 & .043 \\
\hline $\begin{array}{l}\text { Necessity of } \\
\text { library usage } \\
\text { record in } \\
\text { management }\end{array}$ & Necessary & 50 & 61.7 & 39 & 70.9 & 23 & 65.7 & 10 & 71.4 & 10 & 62.5 & .043 \\
\hline $\begin{array}{l}\text { Necessity of } \\
\text { library usage } \\
\text { record in } \\
\text { management }\end{array}$ & Very necessary & 0 & 0 & 0 & 0 & 0 & 0 & 0 & 0 & 0 & 0 & .043 \\
\hline $\begin{array}{l}\text { Necessity of } \\
\text { user consent } \\
\text { in using } \\
\text { library usage } \\
\text { record }\end{array}$ & $\begin{array}{l}\text { Very } \\
\text { unnecessary }\end{array}$ & 0 & 0 & 0 & 0 & 0 & 0 & 0 & 0 & 0 & 0 & .000 \\
\hline $\begin{array}{l}\text { Necessity of } \\
\text { user consent } \\
\text { in using } \\
\text { library usage } \\
\text { record }\end{array}$ & Unnecessary & 4 & 4.9 & 9 & 16.4 & 2 & 5.7 & 2 & 14.3 & 2 & 12.5 & .000 \\
\hline $\begin{array}{l}\text { Necessity of } \\
\text { user consent } \\
\text { in using } \\
\text { library usage } \\
\text { record }\end{array}$ & Neutral & 6 & 7.4 & 7 & 12.7 & 5 & 14.3 & 1 & 7.1 & 0 & 0 & .000 \\
\hline
\end{tabular}


D. S. Kim \& Y. H. Noh

66 International Journal of Knowledge Content Development \& Technology Vol.4, No.1, 53-78 (June, 2014)

\begin{tabular}{|c|c|c|c|c|c|c|c|c|c|c|c|c|}
\hline \multirow{2}{*}{$\begin{array}{l}\text { Item } \\
\text { Item }\end{array}$} & \multirow{2}{*}{$\begin{array}{l}\text { Classification } \\
\text { Classification }\end{array}$} & \multicolumn{2}{|c|}{$\begin{array}{l}\text { Less than } \\
\text { once } \\
\text { a week }\end{array}$} & \multicolumn{2}{|c|}{$\begin{array}{l}\text { Twice } \\
\text { a week }\end{array}$} & \multicolumn{2}{|c|}{$\begin{array}{l}\text { Three } \\
\text { times } \\
\text { a week }\end{array}$} & \multicolumn{2}{|c|}{$\begin{array}{l}\text { Four } \\
\text { times } \\
\text { a week }\end{array}$} & \multicolumn{2}{|c|}{$\begin{array}{l}\text { More than } \\
\text { five times } \\
\text { a week }\end{array}$} & \multirow{2}{*}{$\begin{array}{l}\text { Fisher's } \\
\text { Exact test } \\
\text { Fisher's } \\
\text { Exact test }\end{array}$} \\
\hline & & Freq & $\%$ & Freq & $\%$ & Freq & $\%$ & Freq & $\%$ & Freq & $\%$ & \\
\hline $\begin{array}{l}\text { Necessity of } \\
\text { user consent } \\
\text { in using } \\
\text { library usage } \\
\text { record }\end{array}$ & Necessary & 51 & 63.0 & 35 & 63.6 & 27 & 77.1 & 11 & 78.6 & 13 & 81.3 & .000 \\
\hline $\begin{array}{l}\text { Necessity of } \\
\text { user consent } \\
\text { in using } \\
\text { library usage } \\
\text { record }\end{array}$ & Very necessary & 7 & 8.6 & 4 & 7.3 & 1 & 2.9 & 0 & 0 & 1 & 6.3 & .000 \\
\hline $\begin{array}{l}\text { Necessity of } \\
\text { legal proceeding } \\
\text { when library } \\
\text { usage record is } \\
\text { demanded }\end{array}$ & $\begin{array}{l}\text { Very } \\
\text { unnecessary }\end{array}$ & 0 & 0 & 0 & 0 & 0 & 0 & 0 & 0 & 0 & 0 & .000 \\
\hline $\begin{array}{l}\text { Necessity of } \\
\text { legal proceeding } \\
\text { when library } \\
\text { usage record is } \\
\text { demanded }\end{array}$ & Unnecessary & 2 & 2.5 & 0 & 0 & 2 & 5.7 & 0 & 0 & 1 & 6.3 & .000 \\
\hline $\begin{array}{l}\text { Necessity of } \\
\text { legal proceeding } \\
\text { when library } \\
\text { usage record is } \\
\text { demanded }\end{array}$ & Neutral & 6 & 7.4 & 5 & 9.1 & 4 & 11.4 & 3 & 21.4 & 4 & 25.0 & .000 \\
\hline $\begin{array}{l}\text { Necessity of } \\
\text { legal proceeding } \\
\text { when library } \\
\text { usage record is } \\
\text { demanded }\end{array}$ & Necessary & 54 & 66.7 & 48 & 87.3 & 27 & 77.1 & 10 & 71.4 & 7 & 43.8 & .000 \\
\hline $\begin{array}{l}\text { Necessity of } \\
\text { legal proceeding } \\
\text { when library } \\
\text { usage record is } \\
\text { demanded }\end{array}$ & Very necessary & 6 & 7.4 & 2 & 3.6 & 2 & 5.7 & 1 & 7.1 & 4 & 25.0 & .000 \\
\hline
\end{tabular}

3) Patron understanding of library usage record leaks

A meaningful relationship also seems to exist between frequency of library usage and a greater understanding of the seriousness and causes of library record leaks, as shown in Table 15. 
D. S. Kim \& Y. H. Noh
International Journal of Knowledge Content Development \& Technology Vol.4, No.1, 53-78 (June, 2014)

Table 15. Frequency of Library Use and Records Leak Understanding

\begin{tabular}{|c|c|c|c|c|c|c|c|c|c|c|c|c|}
\hline \multirow{2}{*}{$\begin{array}{l}\text { Item } \\
\text { Item }\end{array}$} & \multirow{2}{*}{$\begin{array}{l}\text { Classification } \\
\text { Classification }\end{array}$} & \multicolumn{2}{|c|}{$\begin{array}{l}\text { Less than } \\
\text { once } \\
\text { a week }\end{array}$} & \multicolumn{2}{|c|}{$\begin{array}{l}\text { Twice } \\
\text { a week }\end{array}$} & \multicolumn{2}{|c|}{$\begin{array}{l}\text { Three times } \\
\text { a week }\end{array}$} & \multicolumn{2}{|c|}{$\begin{array}{l}\text { Four times } \\
\text { a week }\end{array}$} & \multicolumn{2}{|c|}{$\begin{array}{l}\text { More than } \\
\text { fives times } \\
\text { a week }\end{array}$} & \multirow{2}{*}{$\begin{array}{l}\text { Fisher's } \\
\text { Exact test } \\
\text { Fisher's } \\
\text { Exact test }\end{array}$} \\
\hline & & Freq & $\%$ & Freq & $\%$ & Freq & $\%$ & Freq & $\%$ & Freq & $\%$ & \\
\hline $\begin{array}{l}\text { Seriousness of } \\
\text { library usage } \\
\text { record leak }\end{array}$ & Not serious & 0 & 0 & 0 & 0 & 0 & 0 & 0 & 0 & 0 & 0 & .000 \\
\hline $\begin{array}{l}\text { Serious of } \\
\text { library usage } \\
\text { record leak }\end{array}$ & $\begin{array}{l}\text { Not very } \\
\text { serious }\end{array}$ & 11 & 13.6 & 18 & 32.7 & 8 & 22.9 & 4 & 28.6 & 3 & 18.8 & .000 \\
\hline $\begin{array}{l}\text { Serious of } \\
\text { library usage } \\
\text { record leak }\end{array}$ & Neutral & 20 & 24.7 & 10 & 18.2 & 8 & 22.9 & 2 & 14.3 & 4 & 25.0 & .000 \\
\hline $\begin{array}{l}\text { Serious of } \\
\text { library usage } \\
\text { record leak }\end{array}$ & Serious & 32 & 39.5 & 26 & 47.3 & 17 & 48.6 & 8 & 57.1 & 6 & 37.5 & .000 \\
\hline $\begin{array}{l}\text { Serious of } \\
\text { library usage } \\
\text { record leak }\end{array}$ & Very serious & 5 & 6.2 & 1 & 1.8 & 2 & 5.7 & 0 & 0 & 3 & 18.8 & .000 \\
\hline $\begin{array}{l}\text { The cause of } \\
\text { library usage } \\
\text { record leak }\end{array}$ & $\begin{array}{l}\text { Librarian } \\
\text { carelessness }\end{array}$ & 3 & 3.7 & 1 & 1.8 & 2 & 5.7 & 0 & 0 & 0 & 0 & .000 \\
\hline $\begin{array}{l}\text { The cause of } \\
\text { library usage } \\
\text { record leak }\end{array}$ & $\begin{array}{l}\text { Problem with } \\
\text { the computer } \\
\text { system }\end{array}$ & 28 & 34.6 & 20 & 36.4 & 12 & 34.3 & 4 & 28.6 & 2 & 12.5 & .000 \\
\hline $\begin{array}{l}\text { The cause of } \\
\text { library usage } \\
\text { record leak }\end{array}$ & $\begin{array}{l}\text { Patron } \\
\text { carelessness }\end{array}$ & 0 & 0 & 0 & 0 & 2 & 5.7 & 0 & 0 & 0 & 0 & .000 \\
\hline $\begin{array}{l}\text { The cause of } \\
\text { library usage } \\
\text { record leak }\end{array}$ & $\begin{array}{l}\text { Collection of } \\
\text { unnecessary } \\
\text { information }\end{array}$ & 17 & 21.0 & 26 & 47.3 & 8 & 22.9 & 5 & 35.7 & 7 & 43.8 & .000 \\
\hline $\begin{array}{l}\text { The cause of } \\
\text { library usage } \\
\text { record leak }\end{array}$ & $\begin{array}{l}\text { Information } \\
\text { provided to } \\
\text { third parties }\end{array}$ & 20 & 24.7 & 8 & 14.5 & 11 & 31.4 & 5 & 35.7 & 7 & 43.8 & .000 \\
\hline
\end{tabular}

\subsubsection{Understanding of library usage records and level of interest in privacy issues}

1) Understanding of the collection of library usage records

Patron level of interest in privacy issues seemed to be positively correlated with awareness of library record collection and whether or not patrons considered library records to be private information (see Table 16). 
D. S. Kim \& Y. H. Noh

68 International Journal of Knowledge Content Development \& Technology Vol.4, No.1, 53-78 (June, 2014)

Table 16. Interest in Privacy Issues and Library Records Collection

\begin{tabular}{|c|c|c|c|c|c|c|c|c|}
\hline \multirow{2}{*}{$\begin{array}{l}\text { Item } \\
\text { Item }\end{array}$} & \multirow{2}{*}{$\begin{array}{l}\text { Classification } \\
\text { Classification }\end{array}$} & \multicolumn{2}{|c|}{ Interested } & \multicolumn{2}{|c|}{ Neutral } & \multicolumn{2}{|c|}{ Not interested } & \multirow{2}{*}{$\begin{array}{l}\text { Fisher's } \\
\text { Exact test } \\
\text { Fisher's } \\
\text { Exact test }\end{array}$} \\
\hline & & Freq & $\%$ & Freq & $\%$ & Freq & $\%$ & \\
\hline $\begin{array}{l}\text { Awareness of } \\
\text { the collection } \\
\text { of library } \\
\text { usage records }\end{array}$ & Had no clue & 3 & 2.0 & 2 & 7.1 & 0 & 0 & .021 \\
\hline $\begin{array}{l}\text { Awareness of } \\
\text { the collection } \\
\text { of library } \\
\text { usage record }\end{array}$ & Unaware & 75 & 49.3 & 11 & 39.3 & 4 & 50.0 & .021 \\
\hline $\begin{array}{l}\text { Awareness of } \\
\text { the collection } \\
\text { of library } \\
\text { usage record }\end{array}$ & Neutral & 31 & 20.4 & 8 & 28.6 & 2 & 25.0 & .021 \\
\hline $\begin{array}{l}\text { Awareness of } \\
\text { the collection } \\
\text { of library } \\
\text { usage record }\end{array}$ & Knew & 43 & 28.3 & 7 & 25.0 & 2 & 25.0 & .021 \\
\hline $\begin{array}{l}\text { Awareness of } \\
\text { the collection } \\
\text { of library } \\
\text { usage record }\end{array}$ & Knew well & 0 & 0 & 0 & 0 & 0 & 0 & .021 \\
\hline $\begin{array}{l}\text { Recognition of } \\
\text { library usage } \\
\text { data as private } \\
\text { information }\end{array}$ & Never & 0 & 0 & 0 & 0 & 0 & 0 & .000 \\
\hline $\begin{array}{l}\text { Recognition of } \\
\text { library usage } \\
\text { data as private } \\
\text { information }\end{array}$ & No & 15 & 9.9 & 6 & 21.4 & 3 & 37.5 & .000 \\
\hline $\begin{array}{l}\text { Recognition of } \\
\text { library usage } \\
\text { data as private } \\
\text { information }\end{array}$ & Neutral & 28 & 18.4 & 7 & 25.0 & 2 & 25.0 & .000 \\
\hline $\begin{array}{l}\text { Recognition of } \\
\text { library usage } \\
\text { data as private } \\
\text { information }\end{array}$ & Yes & 105 & 69.1 & 14 & 50.0 & 2 & 25.0 & .000 \\
\hline $\begin{array}{l}\text { Recognition of } \\
\text { library usage } \\
\text { data as private } \\
\text { information }\end{array}$ & Very much & 4 & 2.6 & 1 & 3.6 & 1 & 12.5 & .000 \\
\hline
\end{tabular}


D. S. Kim \& Y. H. Noh
International Journal of Knowledge Content Development \& Technology Vol.4, No.1, 53-78 (June, 2014)

2) Understanding of the use of library usage record

Patron level of interest in privacy issues was also correlated with viewing library usage records as necessary for the library's operation and the necessity of legal procedures should these records be requested by a third party (see Table 17).

Table 17. Interest in privacy issues and Use of Library Records

\begin{tabular}{|c|c|c|c|c|c|c|c|c|}
\hline \multirow{2}{*}{$\begin{array}{l}\text { Item } \\
\text { Item }\end{array}$} & \multirow{2}{*}{$\begin{array}{l}\text { Classification } \\
\text { Classification }\end{array}$} & \multicolumn{2}{|c|}{ Interested } & \multicolumn{2}{|c|}{ Neutral } & \multicolumn{2}{|c|}{ Not interested } & \multirow{2}{*}{$\begin{array}{l}\text { Fisher's } \\
\text { Exact test } \\
\text { Fisher's } \\
\text { Exact test }\end{array}$} \\
\hline & & Freq & $\%$ & Freq & $\%$ & Freq & $\%$ & \\
\hline $\begin{array}{l}\text { Necessity of } \\
\text { library usage } \\
\text { records in } \\
\text { management }\end{array}$ & Very unnecessary & 1 & 0.7 & 0 & 0 & 0 & 0 & .000 \\
\hline $\begin{array}{l}\text { Necessity of } \\
\text { library usage } \\
\text { record in } \\
\text { management }\end{array}$ & unnecessary & 2 & 1.3 & 0 & 0 & 1 & 12.5 & .000 \\
\hline $\begin{array}{l}\text { Necessity of } \\
\text { library usage } \\
\text { record in } \\
\text { management }\end{array}$ & Neutral & 36 & 23.7 & 14 & 50.0 & 2 & 25.0 & .000 \\
\hline $\begin{array}{l}\text { Necessity of } \\
\text { library usage } \\
\text { record in } \\
\text { management }\end{array}$ & Necessary & 113 & 74.3 & 14 & 50.0 & 5 & 62.5 & .000 \\
\hline $\begin{array}{l}\text { Necessity of } \\
\text { library usage } \\
\text { record in } \\
\text { management }\end{array}$ & Very necessary & 0 & 0 & 0 & 0 & 0 & 0 & .000 \\
\hline $\begin{array}{l}\text { Necessity of } \\
\text { user consent in } \\
\text { the use of } \\
\text { library usage } \\
\text { records }\end{array}$ & Very unnecessary & 0 & 0 & 0 & 0 & 0 & 0 & .000 \\
\hline $\begin{array}{l}\text { Necessity of } \\
\text { user consent in } \\
\text { the use of } \\
\text { library usage } \\
\text { record }\end{array}$ & unnecessary & 17 & 11.2 & 2 & 7.1 & 0 & 0 & .000 \\
\hline $\begin{array}{l}\text { Necessity of } \\
\text { user consent in } \\
\text { the use of } \\
\text { library usage } \\
\text { record }\end{array}$ & Neutral & 14 & 9.2 & 3 & 10.7 & 2 & 25.0 & .000 \\
\hline
\end{tabular}


D. S. Kim \& Y. H. Noh

70 International Journal of Knowledge Content Development \& Technology Vol.4, No.1, 53-78 (June, 2014)

\begin{tabular}{|c|c|c|c|c|c|c|c|c|}
\hline \multirow{2}{*}{$\begin{array}{l}\text { Item } \\
\text { Item }\end{array}$} & \multirow{2}{*}{$\begin{array}{l}\text { Classification } \\
\text { Classification }\end{array}$} & \multicolumn{2}{|c|}{ Interested } & \multicolumn{2}{|c|}{ Neutral } & \multicolumn{2}{|c|}{ Not interested } & \multirow{2}{*}{$\begin{array}{l}\text { Fisher's } \\
\text { Exact test } \\
\text { Fisher's } \\
\text { Exact test }\end{array}$} \\
\hline & & Freq & $\%$ & Freq & $\%$ & Freq & $\%$ & \\
\hline $\begin{array}{l}\text { Necessity of } \\
\text { user consent in } \\
\text { the use of } \\
\text { library usage } \\
\text { record }\end{array}$ & Necessary & 111 & 73.0 & 21 & 75.0 & 5 & 62.5 & .000 \\
\hline $\begin{array}{l}\text { Necessity of } \\
\text { user consent in } \\
\text { the use of } \\
\text { library usage } \\
\text { record }\end{array}$ & Very necessary & 10 & 6.6 & 2 & 7.1 & 1 & 12.5 & .000 \\
\hline $\begin{array}{l}\text { Necessity of } \\
\text { legal } \\
\text { proceedings } \\
\text { when library } \\
\text { usage records } \\
\text { demanded }\end{array}$ & Very unnecessary & 0 & 0 & 0 & 0 & 0 & 0 & .000 \\
\hline $\begin{array}{l}\text { Necessity of } \\
\text { legal } \\
\text { proceedings } \\
\text { when library } \\
\text { usage record is } \\
\text { demanded }\end{array}$ & unnecessary & 1 & 0.7 & 2 & 7.1 & 2 & 25.0 & .000 \\
\hline $\begin{array}{l}\text { Necessity of } \\
\text { legal } \\
\text { proceedings } \\
\text { when library } \\
\text { usage record is } \\
\text { demanded }\end{array}$ & Neutral & 13 & 8.6 & 6 & 21.4 & 3 & 37.5 & .000 \\
\hline $\begin{array}{l}\text { Necessity of } \\
\text { legal } \\
\text { proceedings } \\
\text { when library } \\
\text { usage record is } \\
\text { demanded }\end{array}$ & Necessary & 127 & 83.6 & 17 & 60.7 & 2 & 25.0 & .000 \\
\hline $\begin{array}{l}\text { Necessity of } \\
\text { legal } \\
\text { proceedings } \\
\text { when library } \\
\text { usage record is } \\
\text { demanded }\end{array}$ & Very necessary & 11 & 7.2 & 3 & 10.7 & 1 & 12.5 & .000 \\
\hline
\end{tabular}

\section{3) Patron understanding of library usage record leaks}

Patron views on the seriousness of library usage record leaks and probable causes of such leaks were also correlated with interest in privacy issues (see Table 18). 
D. S. Kim \& Y. H. Noh

International Journal of Knowledge Content Development \& Technology Vol.4, No.1, 53-78 (June, 2014)

Table 18. Interest in Privacy Issues and Library Record Leaks

\begin{tabular}{|c|c|c|c|c|c|c|c|c|}
\hline \multirow{2}{*}{$\begin{array}{l}\text { Item } \\
\text { Item }\end{array}$} & \multirow{2}{*}{$\begin{array}{l}\text { Classification } \\
\text { Classification }\end{array}$} & \multicolumn{2}{|c|}{ Interested } & \multicolumn{2}{|c|}{ Neutral } & \multicolumn{2}{|c|}{ Uninterested } & \multirow{2}{*}{$\begin{array}{l}\text { Fisher's } \\
\text { Exact test } \\
\text { Fisher's } \\
\text { Exact test }\end{array}$} \\
\hline & & Freq & $\%$ & Freq & $\%$ & Freq & $\%$ & \\
\hline $\begin{array}{l}\text { Seriousness of } \\
\text { library usage } \\
\text { record leaks }\end{array}$ & Not serious & 0 & 0 & 0 & 0 & 0 & 0 & .000 \\
\hline $\begin{array}{l}\text { Seriousness of } \\
\text { library usage } \\
\text { record leaks }\end{array}$ & Not very serious & 34 & 22.4 & 7 & 25.0 & 3 & 37.5 & .000 \\
\hline $\begin{array}{l}\text { Seriousness of } \\
\text { library usage } \\
\text { record leaks }\end{array}$ & Neutral & 28 & 18.4 & 13 & 46.4 & 3 & 37.5 & .000 \\
\hline $\begin{array}{l}\text { Seriousness of } \\
\text { library usage } \\
\text { record leaks }\end{array}$ & Serious & 81 & 53.3 & 7 & 25.0 & 1 & 12.5 & .000 \\
\hline $\begin{array}{l}\text { Seriousness of } \\
\text { library usage } \\
\text { record leaks }\end{array}$ & Very serious & 9 & 5.9 & 1 & 3.6 & 1 & 12.5 & .000 \\
\hline $\begin{array}{l}\text { The cause of } \\
\text { library usage } \\
\text { record leaks }\end{array}$ & $\begin{array}{l}\text { Librarian } \\
\text { carelessness }\end{array}$ & 3 & 2.0 & 2 & 7.1 & 1 & 12.5 & .000 \\
\hline $\begin{array}{l}\text { The cause of } \\
\text { library usage } \\
\text { record leaks }\end{array}$ & $\begin{array}{l}\text { Problems with } \\
\text { the computer } \\
\text { system }\end{array}$ & 55 & 36.2 & 9 & 32.1 & 2 & 25.0 & .000 \\
\hline $\begin{array}{l}\text { The cause of } \\
\text { library usage } \\
\text { record leaks }\end{array}$ & $\begin{array}{l}\text { Patron } \\
\text { carelessness }\end{array}$ & 2 & 1.3 & 0 & 0 & 0 & 0 & .000 \\
\hline $\begin{array}{l}\text { The cause of } \\
\text { library usage } \\
\text { record leaks }\end{array}$ & $\begin{array}{l}\text { Collection of } \\
\text { unnecessary } \\
\text { information }\end{array}$ & 56 & 36.8 & 5 & 17.9 & 2 & 25.0 & .000 \\
\hline $\begin{array}{l}\text { The cause of } \\
\text { library usage } \\
\text { record leaks }\end{array}$ & $\begin{array}{l}\text { Information } \\
\text { provided to } \\
\text { third parties }\end{array}$ & 36 & 23.7 & 12 & 42.9 & 3 & 37.5 & .000 \\
\hline
\end{tabular}

\subsubsection{Correlation between damage caused to patrons by private information leak and library usage record understanding}

1) Understanding of the collection of library usage record

No meaningful relationship existed in this survey between patrons' past experience with damage from a record leak and their understanding of the collection of usage records by libraries (see Table 19). 
72 International Journal of Knowledge Content Development \& Technology Vol.4, No.1, 53-78 (June, 2014)

Table 19. Past Leak Experience and Library Records Collection

\begin{tabular}{|c|c|c|c|c|c|c|}
\hline Item & Classification & $\begin{array}{l}\text { Expe } \\
\text { dama }\end{array}$ & & $\begin{array}{l}\text { Neve } \\
\text { dama }\end{array}$ & iced & $\begin{array}{l}\text { Fisher's } \\
\text { Exact test }\end{array}$ \\
\hline Item & Classification & Freq & $\%$ & Freq & $\%$ & $\begin{array}{l}\text { Fisher's } \\
\text { Exact test }\end{array}$ \\
\hline $\begin{array}{l}\text { Awareness of } \\
\text { the collection of } \\
\text { library usage } \\
\text { records }\end{array}$ & Had no clue & 0 & 0 & 5 & 3.1 & .298 \\
\hline $\begin{array}{l}\text { Awareness of } \\
\text { the collection of } \\
\text { library usage } \\
\text { record }\end{array}$ & Unaware & 10 & 35.7 & 80 & 50.0 & .298 \\
\hline $\begin{array}{l}\text { Awareness of } \\
\text { the collection of } \\
\text { library usage } \\
\text { record }\end{array}$ & Neutral & 8 & 28.6 & 33 & 20.6 & .298 \\
\hline $\begin{array}{l}\text { Awareness of } \\
\text { the collection of } \\
\text { library usage } \\
\text { record }\end{array}$ & Knew & 10 & 35.7 & 42 & 26.3 & .298 \\
\hline $\begin{array}{l}\text { Awareness of } \\
\text { the collection of } \\
\text { library usage } \\
\text { record }\end{array}$ & Knew well & 0 & 0 & 0 & 0 & .298 \\
\hline $\begin{array}{l}\text { Realization of } \\
\text { library usage } \\
\text { records as private } \\
\text { information }\end{array}$ & Never & 0 & 0 & 0 & 0 & .080 \\
\hline $\begin{array}{l}\text { Realization of } \\
\text { library usage } \\
\text { record as private } \\
\text { information }\end{array}$ & No & 0 & 0 & 24 & 15.0 & .080 \\
\hline $\begin{array}{l}\text { Realization of } \\
\text { library usage } \\
\text { record as private } \\
\text { information }\end{array}$ & Neutral & 6 & 21.4 & 31 & 19.4 & .080 \\
\hline $\begin{array}{l}\text { Realization of } \\
\text { library usage } \\
\text { record as private } \\
\text { information }\end{array}$ & Yes & 21 & 75.0 & 100 & 62.5 & .080 \\
\hline $\begin{array}{l}\text { Realization of } \\
\text { library usage } \\
\text { record as private } \\
\text { information }\end{array}$ & Very much & 1 & 3.6 & 5 & 3.1 & .080 \\
\hline
\end{tabular}

\section{2) Understanding of the use of library usage record}

Patrons' past experience with leaked information did have a meaningful relationship with their views of the necessity of usage records for library operation. However, past experience did not correlate with patron demands for consent before using library records or the necessity of legal procedures for when records are requested by a third party. 
D. S. Kim \& Y. H. Noh
International Journal of Knowledge Content Development \& Technology Vol.4, No.1, 53-78 (June, 2014)

Table 20. Past Record Leak Experience and Library Record Use

\begin{tabular}{|c|c|c|c|c|c|c|}
\hline \multirow{2}{*}{$\begin{array}{l}\text { Item } \\
\text { Item }\end{array}$} & \multirow{2}{*}{$\begin{array}{l}\text { Classification } \\
\text { Classification }\end{array}$} & \multicolumn{2}{|c|}{ Experienced damage } & \multicolumn{2}{|c|}{ No damage experienced } & \multirow{2}{*}{$\begin{array}{l}\text { Fisher's } \\
\text { Exact test } \\
\text { Fisher's } \\
\text { Exact test }\end{array}$} \\
\hline & & Freq & $\%$ & Freq & $\%$ & \\
\hline $\begin{array}{l}\text { Necessity of library } \\
\text { usage records for } \\
\text { management }\end{array}$ & Very unnecessary & 0 & 0 & 1 & 0.6 & .032 \\
\hline $\begin{array}{l}\text { Necessity of library } \\
\text { usage record for its } \\
\text { management }\end{array}$ & Unnecessary & 2 & 7.1 & 1 & 0.6 & .032 \\
\hline $\begin{array}{l}\text { Necessity of library } \\
\text { usage record for its } \\
\text { management }\end{array}$ & Neutral & 9 & 32.1 & 43 & 26.9 & .032 \\
\hline $\begin{array}{l}\text { Necessity of library } \\
\text { usage record for its } \\
\text { management }\end{array}$ & Necessary & 17 & 60.7 & 115 & 71.9 & .032 \\
\hline $\begin{array}{l}\text { Necessity of library } \\
\text { usage record for its } \\
\text { management }\end{array}$ & Very necessary & 0 & 0 & 0 & 0 & .032 \\
\hline $\begin{array}{l}\text { Necessity of user } \\
\text { consent in using } \\
\text { library usage records }\end{array}$ & Very unnecessary & 0 & 0 & 0 & 0 & .154 \\
\hline $\begin{array}{l}\text { Necessity of user } \\
\text { consent in using } \\
\text { library usage record }\end{array}$ & Unnecessary & 1 & 3.6 & 18 & 11.3 & .154 \\
\hline $\begin{array}{l}\text { Necessity of user } \\
\text { consent in using } \\
\text { library usage record }\end{array}$ & Neutral & 3 & 10.7 & 16 & 10.0 & .154 \\
\hline $\begin{array}{l}\text { Necessity of user } \\
\text { consent in using } \\
\text { library usage record }\end{array}$ & Necessary & 20 & 71.4 & 117 & 73.1 & .154 \\
\hline $\begin{array}{l}\text { Necessity of user } \\
\text { consent in using } \\
\text { library usage record }\end{array}$ & Very necessary & 4 & 14.3 & 9 & 5.6 & .154 \\
\hline $\begin{array}{l}\text { Necessity of legal } \\
\text { procedures when } \\
\text { library usage records } \\
\text { demanded }\end{array}$ & Very unnecessary & 0 & 0 & 0 & 0 & .388 \\
\hline $\begin{array}{l}\text { Necessity of legal } \\
\text { procedures when } \\
\text { library usage record } \\
\text { is demanded }\end{array}$ & Unnecessary & 1 & 3.6 & 4 & 2.5 & .388 \\
\hline $\begin{array}{l}\text { Necessity of legal } \\
\text { procedures when } \\
\text { library usage record } \\
\text { is demanded }\end{array}$ & Neutral & 3 & 10.7 & 19 & 11.9 & .388 \\
\hline $\begin{array}{l}\text { Necessity of legal } \\
\text { procedures when } \\
\text { library usage record } \\
\text { is demanded }\end{array}$ & Necessary & 20 & 71.4 & 126 & 78.8 & .388 \\
\hline $\begin{array}{l}\text { Necessity of legal } \\
\text { procedures when } \\
\text { library usage record } \\
\text { is demanded }\end{array}$ & Very necessary & 44 & 14.3 & 11 & 6.9 & .388 \\
\hline
\end{tabular}


D. S. Kim \& Y. H. Noh

74 International Journal of Knowledge Content Development \& Technology Vol.4, No.1, 53-78 (June, 2014)

3) Understanding leaks of library usage records

No correlation existed between patrons' past experience with record leaks and their views on the seriousness of record leaks. However, a meaningful relationship did exist between past experience and patron-selected probable causes of records leaks.

Table 21. Past Records Leak Experience and Library Records Leak Understanding

\begin{tabular}{|c|c|c|c|c|c|c|}
\hline \multirow{2}{*}{$\begin{array}{l}\text { Item } \\
\text { Item }\end{array}$} & \multirow{2}{*}{$\begin{array}{l}\text { Classification } \\
\text { Classification }\end{array}$} & \multicolumn{2}{|c|}{ Experienced damage } & \multicolumn{2}{|c|}{ No damage experienced } & \multirow{2}{*}{$\begin{array}{l}\text { Fisher } \\
\text { Exact test } \\
\text { Fisher } \\
\text { Exact test }\end{array}$} \\
\hline & & Freq & $\%$ & Freq & $\%$ & \\
\hline $\begin{array}{l}\text { Seriousness of } \\
\text { library usage } \\
\text { record leaks }\end{array}$ & Not serious & 0 & 0 & 0 & 0 & .213 \\
\hline $\begin{array}{l}\text { 성Seriousness of } \\
\text { library usage } \\
\text { record leaks }\end{array}$ & Not very serious & 3 & 10.7 & 41 & 25.6 & .213 \\
\hline $\begin{array}{l}\text { 성Seriousness of } \\
\text { library usage } \\
\text { record leaks }\end{array}$ & Neutral & 6 & 21.4 & 38 & 23.8 & .213 \\
\hline $\begin{array}{l}\text { 성Seriousness of } \\
\text { library usage } \\
\text { record leaks }\end{array}$ & Serious & 17 & 60.7 & 72 & 45.0 & .213 \\
\hline $\begin{array}{l}\text { 성Seriousness of } \\
\text { library usage } \\
\text { record leaks }\end{array}$ & Very serious & 2 & 7.1 & 9 & 5.6 & .213 \\
\hline $\begin{array}{l}\text { Cause of library } \\
\text { information } \\
\text { leaks }\end{array}$ & $\begin{array}{l}\text { Librarian } \\
\text { carelessness }\end{array}$ & 1 & 3.6 & 5 & 3.1 & .000 \\
\hline $\begin{array}{l}\text { The cause of } \\
\text { library information } \\
\text { leaks }\end{array}$ & $\begin{array}{l}\text { Computer } \\
\text { system problem }\end{array}$ & 14 & 50.0 & 52 & 32.5 & .000 \\
\hline $\begin{array}{l}\text { The cause of } \\
\text { library information } \\
\text { leaks }\end{array}$ & $\begin{array}{l}\text { Patron } \\
\text { carelessness }\end{array}$ & 0 & 0 & 2 & 1.3 & .000 \\
\hline $\begin{array}{l}\text { The cause of } \\
\text { library information } \\
\text { leaks }\end{array}$ & $\begin{array}{l}\text { Collection of } \\
\text { unnecessary } \\
\text { information }\end{array}$ & 1 & 3.6 & 62 & 38.8 & .000 \\
\hline $\begin{array}{l}\text { The cause of } \\
\text { library information } \\
\text { leaks }\end{array}$ & $\begin{array}{l}\text { Information } \\
\text { provided to } \\
\text { third parties }\end{array}$ & 12 & 42.9 & 39 & 24.4 & .000 \\
\hline
\end{tabular}




\section{Conclusion and proposal}

The importance of protecting private information is becoming ever more important in all aspects of society, and therefore should be of great interest to libraries as well. After the privacy protection act was established, many studies focused on the collection and preservation of data that the library gathers about its patrons. However, However, no research exists from the users' perspective, even though their views are perhaps the most important to consider, since they are most affected by policies created to address these issues. The purpose of researching the comprehensive understanding of library patrons about these issues is not because current personal information management policies do not meet the requirements set forth in law, but rather to offer a policy that satisfies the protection of the patron's idea of intellectual freedom and privacy. Because the library needs patrons to fulfill its purpose, it must ensure them these two rights. This study researched the library patrons' understanding of private information in order to form an effective personal information management policy.

1) Comprehensive understanding of library usage records

First, $50.6 \%$ of library patrons were unaware that their library was collecting this data about them, and $64.4 \%$ perceived library usage records to be private information. Among the records, the material borrowing records were considered the most important, before even Internet usage history and CCTV footage.

Second, results showed that in the use of library records, $70.2 \%$ of respondents understood the necessity of the records for the library's effective operation. It can be assumed from this evidence that patrons could easily make the connection between the kinds of records kept and the services offered to them by their libraries. A majority of respondents $(79.8 \%)$ thought patron approval was necessary for the use of library records and $85.7 \%$ responded that legal procedures were necessary for outsiders or third parties such as police or other government officials to view their records.

Third, $53.2 \%$ of patrons thought library usage record leaks were serious, with $35.1 \%$ choosing the computer system as the most likely source for such leaks. However, other possibilities of leak sources also had similar rates of response, such as the collection of unnecessary information (33.5\%) and information provided to third parties (27.1\%).

2) Relationship between personal characteristics of patrons and understanding of library usage records

We examined possible correlations between different patron characteristics that were posited to be relevant to this discussion (frequency of library use, interest in privacy issues, and past experience as the victim of an information leak) and the results of the survey questions about library records.

As a result, frequent visits to the library and patron interest in privacy issue both had meaningful correlations in relation to patron understanding of the collection, use, and possible leaks of library usage records.

Past experience with being the victim of a privacy violation or information leak was found to have no meaningful correlation with the understanding of the collection, use, and possible leaks of library records. While meaningful relationships did exist between this background characteristic 
and some aspects of the three surveyed categories, there was no measurable overall effect on any of the three main aspects.

With these results, the private information management plan that this study suggests in as follows. First, patrons should be more clearly informed about the information that is collected and used at the library when they first sign-up for a library card. In this study, the patrons surveyed replied that they were not aware that the library collected and preserved their usage records. Even though many libraries specify that they collect, preserve, and use the patron's library usage data in the sign-up terms of service consent form, both online and office, this finding means that most patrons do not carefully read the terms. Also, these results show that, though patrons think that the collection, preservation, and use of their library records are necessary for library operations, they still should be asked for consent before these activities commence.

After the enactment of the privacy protection act, as a strategy to decrease the amount of documents containing private information, many libraries changed their sign-up process so that it could only be accomplished online. However, libraries did not provide an easily understandable online terms and conditions notice to users signing up in this manner. Considering the characteristics of a public library, where various age and socio-economic groups are all served, it is important to provide a terms of service notice during the initial sign-up that all users can easily understand. This wider knowledge would ensure that all user rights are protected when using the library.

Second, library networks should be monitored for possible leaks, and when one occurs, its source should be determined immediately. This study shows that patrons think there are a few possible causes for such leaks, especially, computer system problems and private information provided to third parties, which hint that leaks of library information can come from both inside and outside of the institution.

Therefore, continuous education regarding privacy issues must be given to librarians in order for library staff to prevent but also be ready in case of a privacy leak. The importance of protecting private information should also be stressed to staff. Also, considering that most of the library's private information is preserved in a digital format, a systematic maintenance manual for computer systems must be developed.

Through this study, one can see that a meaningful relationship exists between the frequency of library use, interest in privacy issues, and the comprehensive understanding of library usage records. However, a follow-up study is necessary because this preliminary research was only purposed to determine if the relationship was true or not.

First, the patrons' comprehensive understanding of library records was studied, with the target group not having any previous education on the importance of private information. In future studies, a comparison of the comprehensive understanding of privacy information study from many different angles is necessary. Second, the gap in comprehensive understanding between the librarian managing the library and its patrons must be evaluated as well as research to see how much the comprehensive understanding of the librarian and patrons affect the private information management policies in each existing library. Third, along with determining if there is an information protection policy in each library regarding the library usage record, a study regarding the protection of library usage records must be made. 
D. S. Kim \& Y. H. Noh

International Journal of Knowledge Content Development \& Technology Vol.4, No.1, 53-78 (June, 2014)

\section{References}

Balas, J. (2001). How should privacy be protected in the electronic library. Computers in Libraries, 21(6), 53-55.

Bower, S. L. (2006). Privacy and library records. Journal of Academic Librarianship, 32(4), 377-383.

Bower, S. L. (2008). Self-service holds: A violation of library patron's privacy. Public Libraries, 47(4), 54-57.

Breedingm M. (2006). Technology for the next generation. Computers in Libraries, 26(10), 28-30.

Butters, A. (2007). RFID systems, standards and privacy within libraries. The Electronic Library, 25(4), 430-439.

Caldwell-Stone, D. (2008). Privacy, libraries, and ALA American Libraries, September, 58.

Clarke, R. (1996). Privacy and dataveillance, and organisational strategy. Keynote address to the Conference of the I.S. Audit \& Control Association. Perth, Western Australia.

DeMarco, D. A. (2006). Understanding consumer information privacy in the realm of Internet cinnerce: Personhood and pragmatism, pop tarts and six packs. Texas Law Review, 87(11), 1013-1017.

Enright, K. P. (2001). Privacy audit checklist. Retrieved from http://cyber.law.harvard.edu/clinical/priva cyaudit.html

Falk, Howard. (2004). Privacy in libraries. The Electronic Library, 22(3), 281-284.

Fifarek, A. (2002). Technology and privacy in the academic library. Online Information Review, 26(6). 366-374.

Johnston, S. D. (2000). Rethinking privacy in the public library. The International Information \& Library Review, 32(3-4), 509-517.

Joung, Hyun-Tae. (2000). A study on the intellectual freedom in Korean public libraries. Journal of the Korean Society for Library and Information Science, 34(1), 243-264.

Kang Sun Hui. (2003). Saseo mit jeongbo service jikui yunli uisik-e gwanhan yeongu. Library with People, 4(4), 53-55.

Kennedy, S. D. (2006). I've been violated. Information Today. 17. 20.

Kim, Gi-Mun. (2003). Miguk-ui aegukbeobgwa doseogwan iyoungjaui privacy. Library with People, $4(4), 60-71$.

Kim, Hae-Kyoung, \& Nam, Tae-Woo. (2004). A new model for codes of ethics for librarians of South Korea. Journal of the Korean Society for Information Management, 21(4), 329-352.

Kim, Hye-Sun. (1994). A Study on Ethics in Librarianship. M.A. thesis, Ewha Womans University.

Kim, Ki-Seong. (2006). A Study on the Privacy of University Libraries. M.A. thesis, Chungnam University.

Kim, Song-soo. (2012). A study on library user privacy protection in the digital environment: Focused on record of checked out books. Hanyang University, Graduate School of Public Policy.

Klinefelter, A. (2007). Privacy and library public services: Or, I know what you read last summer. Legal Reference Serivces Quarterly, 26(1-2), 253-279.

Lee, Mi-Hwa. (2006). A study on user perceptions about privacy and library records. The Journal of the Korean Private University Library Association, 7, 225-240.

Lee, Myeong-Hee. (2004). Libraries' efforts to preserve intellectual freedom and banned book. Journal 
D. S. Kim \& Y. H. Noh
International Journal of Knowledge Content Development \& Technology Vol.4, No.1, 53-78 (June, 2014)

of the Korean Biblia Society for library and Information Science, 15(1), 193-216.

Leiserson, A. B. (2002). A user's perspective on privacy and the web. Law Library Journal, 94(3), 539-546.

Levine, C. (2006). IM: Real-time problems: Traders increasingly are adopting public instant messaging services, but most of these applications open severe security goles. Wall Street \& Technology.

Noh, Younghee. (2012a). A Study on Developing and Proposing the Library Privay Policy. Journal of Korean Library and Information Science Society, 46(4), 207-242.

Noh, Younghee. (2012b). A study of personalized user services and privay in the library. Journal of Korean Library and Information Science Society, 43(3), 357-398.

Noh, Younghee. (2012c). A study of digital library service records and user privacy. Journal of the Korean Society for Information Management, 29(3), 187-214.

Noh, Younghee. (2013). A study on librariands perception of library user privacy. Journal of Korean Library and Information Science Society, 47(3), 73-96.

Noh, Younghee. (2013). Digital Library User privacy: Changing Librarian View points through Education. in progress.

OECD. (1980). OECD Guidelines on the Protection of Privacy and Transborder Flows of Personal Data [online] <http://www.oecd.org/sti/ieconomy/oecdguidelinesontheprotectionofprivacyandtransborderflowsofpersonaldata.htm\#part3 $>$ Retrieved 2013.08.23.

Park, Sang-Keun. (2010). A study on the plans for the reinforcement of the personal information protection policy in public libraries. Graduate School of Kyonggi University, Department of Library and Information Science.

Shuler, J. (2004). Privacy and academic libraries: Widening the frame of discussion. Journal of Academic Librarianship, 30(2), 157-159.

Sturges, P. (2002). Remember the human: The first rule of netquette, librarians and the internet. Online Information Review, 26(3), 209-16.

The National Library of Korea. (2012). Guidelines for personal information protection. Seoul: The National Library of Korea.

Tripathi, Sneha, \& Tripathi, Aditya. (2010). Privacy in libraries: The Perspective from India. Library Review, 59(8), 615-623.

Wallace, M. (1988). Ethics: Is it time for a code? In managing the law firm library 1988. 258 PIL/ PAT 329(Sept. 1, 1988).

Wareen, S. D., \& Brandeis, L. D. (1890). The right to privacy. Harvard Law Review. 4, 193.

Zimerman, M. (2009). Technology and privacy erosion in united states libraries: A personal viewpoint. New Library World, 111(1/2), 7-15. 